\title{
In-Situ Monitoring of Chemical Vapor Deposition from Trichlorosilane Gas and Monomethylsilane Gas Using Langasite Crystal Microbalance
}

\author{
Hitoshi Habuka, Yurie Tanaka \\ Department of Chemical and Energy Engineering, Yokohama National University, Yokohama, Japan. \\ Email: habuka1@ynu.ac.jp
}

Received October $6^{\text {th }}, 2012$; revised November $10^{\text {th }}, 2012$; accepted November $17^{\text {th }}, 2012$

\begin{abstract}
Using the langasite crystal microbalance (LCM), the trends in film thickness produced by means of the chemical vapor deposition using trichlorosilane gas, monomethylsilane gas and their mixed gas were observed at $600^{\circ} \mathrm{C}$ and evaluated by comparison with the information from a transmission electron microscope (TEM). The crystalline silicon film thickness from trichlorosilane gas was comparable to that of an amorphous silicon carbide film from monomethylsilane gas. The film obtained from the gas mixture was amorphous and was the thinnest in this study. Because the thickness trend obtained by the LCM agreed with that by the TEM, the LCM is shown to be a convenient evaluation tool for the behavior of various film deposition.
\end{abstract}

Keywords: Chemical Vapor Deposition; In-Situ Measurement; Langasite Crystal Microbalance

\section{Introduction}

Chemical vapor deposition (CVD) [1,2] produces valuable material films from various precursors, such as silanes, chlorosilanes, methylsilanes, and organic metals. The film deposition rate is obtained and evaluated using various data for the film, such as thickness, weight and an interferogram, which are very often obtained ex-situ. In order to understand the details of the film formation in a steady state and in a non-steady state, an in-situ measurement is desired.

For performing the in-situ monitoring, the langasite crystal microbalance (LCM) has been evaluated by many researchers [3-6] as a useful tool, because it could be very sensitive to various changes caused by the transport phenomena in the CVD reactor and the film deposition at an order of nanometers/min [5,6]. Additionally, the other advantage of the in-situ monitoring is the capability for quickly evaluating the film deposition trends under various CVD conditions without using a substrate. For extending and pursuing the LCM capability in the CVD study, deposited films having various surface morphologies from various precursors should be measured and evaluated. Additionally, the obtained results should be evaluated by some classical tools, such as the transmission electron microscope (TEM).

For this purpose, an interesting issue is the change in the film deposition process caused by various precursors and their mixture. When a mixture of precursors is introduced into the reactor, the relationship between the total film deposition behavior and that predicted by each precursor may give future motivation for exploring and developing new CVD chemistry.

From this viewpoint, the film deposition behavior of silicon and silicon carbide is an interesting example. Silicon film from trichlorosilane tends to show a crystalline form over a very wide temperature range $[7,8]$. In contrast to this, a silicon carbide film tends to be amorphous at low temperatures [9]. When the two different kinds of film growth are simultaneously performed, the obtained trends and behavior are expected to indicate some information on the surface process.

In this study using the CVD reactor, the film deposition of silicon, silicon carbide and their mixture from the precursors of trichlorosilane and monomethylsilane was in-situ examined by the LCM. The trend of the film thickness was verified, compared with the information obtained by the TEM. The surface chemical process influenced by the mixed precursors was additionally discussed.

\section{Experimental}

In order to perform the film deposition by the CVD me- 
thod, the horizontal cold wall reactor having the LCM, shown in Figure 1, was used. This reactor consists of a gas supply system, a quartz chamber and infrared lamps. The gas supply system can introduce gases, such as hydrogen, nitrogen, trichlorosilane and monomethylsilane. Hydrogen is the carrier gas, the flow rate of which was typically $1 \mathrm{slm}$ at atmospheric pressure. The gas flow channel of this reactor has a low height and a small rectangular cross section in order to achieve a high consumption efficiency of the reactive gases. The height and the width of the quartz chamber were $10 \mathrm{~mm}$ and $40 \mathrm{~mm}$, respectively, similar to those used in our previous studies $[5,6,10-13]$.

A 30-mm-wide $\times 40$-mm-long (100) silicon wafer manufactured by the Czochralski method was horizontally placed on the bottom wall of the quartz chamber. The LCM (Halloran Electronics, Tokyo, Japan) was placed 5 $\mathrm{mm}$ above the silicon wafer and was connected to a personal computer in order to measure its frequency.

The LCM has an intrinsic frequency of $10 \mathrm{MHz}$ similar to our previous studies $[5,6]$. The silicon wafer and the LCM were simultaneously heated by infrared light from halogen lamps through the quartz chamber. The LCM was heated not only by the infrared light but also by radiation heat and conduction heat from the hot silicon wafer. The temperature of the silicon wafer was measured prior to the CVD process using thermocouples. Because the position of the LCM was very near the silicon wafer, the temperature of the LCM was assumed to be the same as that of the silicon wafer.

A typical process used in this study is shown in Figure 2. First, the LCM was heated to $600^{\circ} \mathrm{C}$ in ambient hydrogen. After waiting until the LCM frequency became stable, the trichlorosilane gas and monomethylsilane gas were introduced into the reactor chamber. The flow rate of trichlorosilane gas and monomethylsilane gas was typically $0.01-0.06 \mathrm{slm}$. The temperature of $600^{\circ} \mathrm{C}$ was expected to be the temperature near the lowest possible

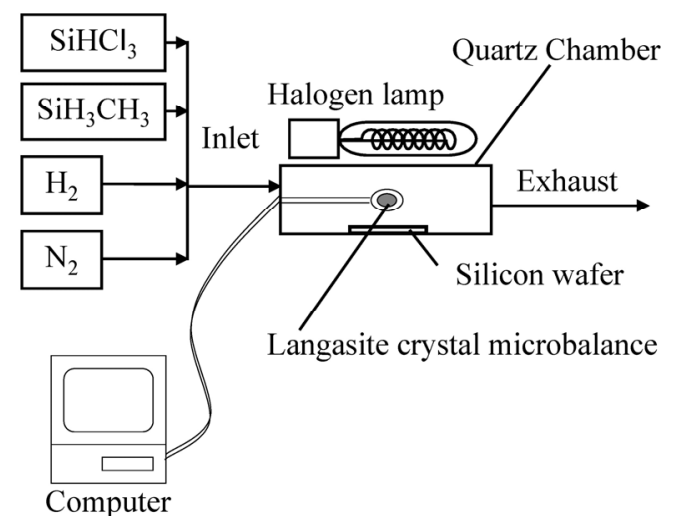

Figure 1. Chemical vapor deposition reactor having a langasite crystal microbalance.

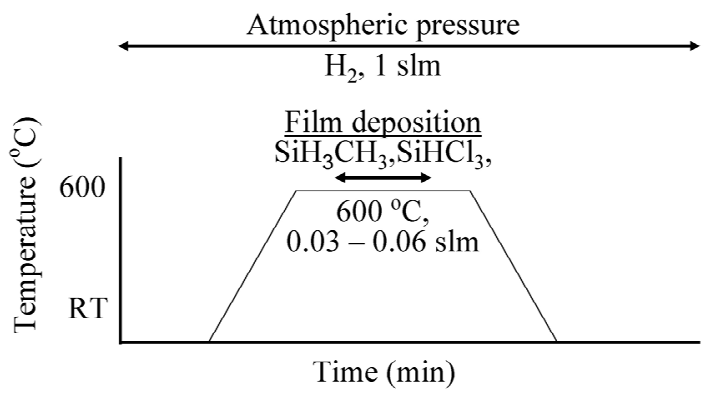

Figure 2. Film deposition process used in this study.

value for the film deposition from the two precursors.

During the introduction of the precursors, the decrease in LCM frequency indicates the weight increase due to the film deposition on the LCM surface. This relationship has been known to follow the Sauerbrey equation [14]. Similar to the previous studies [3-6], the LCM used in this study was assumed to show the relationship of Equation (1) between the frequency decrease, $\Delta f(\mathrm{~Hz})$, the electrode area, $A\left(\mathrm{~cm}^{2}\right)$, and the elastic mass change, $\Delta m$ (ng), at room temperature and high temperatures.

$$
\Delta m /(\Delta f A)=-6.0 \mathrm{ng} /\left(\mathrm{Hz} \cdot \mathrm{cm}^{2}\right)
$$

The electrode of the LCM was made of iridium, because it is inert to various chemicals, including the gases used in this study. Prior to the measurement, no frequency change was detected when it was kept in ambient hydrogen for over several minutes at $700^{\circ} \mathrm{C}$. Thus, the LCM frequency change obtained in this study was not due to electrode corrosion. Prior to measuring the film deposition, the electrode surface was covered with a silicon carbide film using monomethylsilane gas at high temperatures $[12,13]$.

In order to evaluate and verify the results obtained by the LCM, the film deposition was performed on a silicon surface using the same condition as that performed on the LCM. In this study, the native oxide film existing on the silicon surface was not removed in order to employ a surface condition similar to that of the LCM, which might involve fatal damage due to heating at high temperatures for removing the native oxide.

\section{Results and Discussion}

\subsection{In-Situ Measurement by LCM}

Figure 3 shows the behavior of LCM frequency when trichlorosilane gas, monomethylsilane gas and their mixture were introduced into the reactor at $600^{\circ} \mathrm{C}$. For the silicon film deposition, the flow rate of trichlorosilane gas was $0.06 \mathrm{slm}$. The silicon carbide film was formed by monomethylsilane gas at the flow rate of $0.05 \mathrm{slm}$. For performing the film deposition by the gas mixture, the trichlorosilane gas and monomethylsilane gas flow 


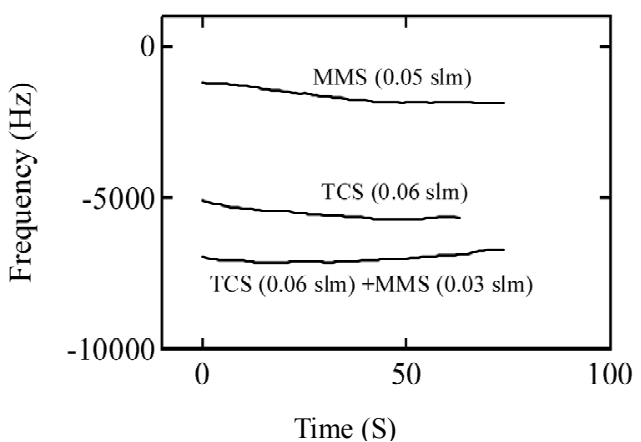

Figure 3. Behavior of LCM frequency during the supply of trichlorosilane gas at the flow rate of $0.06 \mathrm{slm}$, monomethylsilane gas at $0.05 \mathrm{slm}$ and the mixture of trichlorosilane and monomethylsilane at the flow rates of 0.06 and $0.03 \mathrm{slm}$, respectively. Total gas flow rate was $1 \mathrm{slm}$.

rate was set at 0.06 and $0.03 \mathrm{slm}$, respectively.

In Figure 3, the LCM frequency is shown as the frequency difference from that before initiating the precursor supply. When the precursor was supplied, the LCM frequency immediately decreased from that before supplying the precursor. This immediate decrease was caused by the change in the fluid property [6], from the hydrogen ambient to the ambient of hydrogen with precursor gases.

When the silicon film deposition was performed with trichlorosilane gas at the flow rate of $0.06 \mathrm{slm}$, the LCM frequency gradually decreased. This trend also appeared when the silicon carbide film was formed from the monomethylsilane gas at the flow rate of $0.05 \mathrm{slm}$. The decreasing rate of the LCM frequency using the monomethylsilane gas seemed to be comparable to that using the trichlorosilane gas.

Next, the gas mixture of trichlorosilane and monomethylsilane was introduced to the LCM. As shown in Figure 3, the decreasing rate of LCM frequency was very small. This indicates that the weight increase due to the film deposition from the mixture of trichlorosilane gas and monomethylsilane gas was clearly smaller than that with each gas. This behavior is expected to include important information on the change in the film deposition process and the film quality.

\subsection{Film Thickness and Quality by TEM}

Next, the film quality obtained from each precursor and from the mixture was evaluated using the TEM. Figure 4 shows the TEM image of the silicon thin film obtained from the trichlorosilane gas at the flow rate of $0.06 \mathrm{slm}$ in hydrogen gas at $0.94 \mathrm{slm}$, at $600^{\circ} \mathrm{C}$ for $10 \mathrm{~min}$. Figure 4(a) shows the entire surface morphology of the obtained film. This figure shows that the silicon film surface had many sharp hillocks, the maximum height of which was about $20 \mathrm{~nm}$. Figure 4(b) is the magnifica-

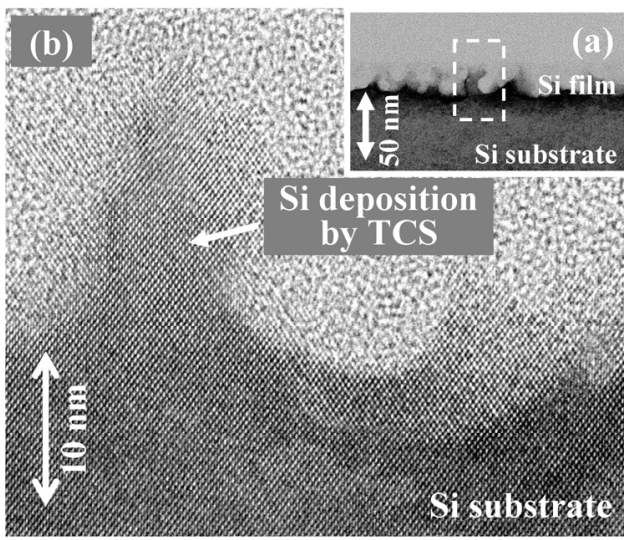

Figure 4. TEM image of silicon thin film obtained from trichlorosilane gas at the flow rate of $0.06 \mathrm{slm}$ in hydrogen gas at $0.94 \mathrm{slm}$ at $600^{\circ} \mathrm{C}$ for $10 \mathrm{~min}$.

tion of the area indicated using the dotted line in Figure 4(a). In Figure 4(b), the small dark dots were the silicon atoms. Because the silicon atoms were regularly arranged from the bottom of this figure to the top of the hillocks, the obtained film was in a crystalline form. Because the film surface was very rough, as shown in Figure 4(a), the average film thickness could be significantly smaller than the top height of the hillocks.

Although the native oxide film at the silicon substrate surface was not removed, the native oxide layer was not clearly observed in the obtained film. Taking into account the influence of the remaining native oxide film, the steep hillocks might be caused at the spots which had a chance of native oxide removal by any means, such as cleavage or collapse at the early stage of the film deposition.

Figure 5 shows the TEM image of a silicon carbide thin film obtained from the monomethylsilane gas at the flow rate of $0.05 \mathrm{slm}$ in hydrogen gas at $0.95 \mathrm{slm}$ at $600^{\circ} \mathrm{C}$ for $10 \mathrm{~min}$. Figure 5(a) shows the entire surface morphology of the obtained film. Taking into account the weak contrast to the non-solid region, the silicon carbide film thickness could be estimated to be about $5 \mathrm{~nm}$. Additionally, the film surface showed a flat and smooth morphology. Figure 5(b) is a magnification of the area indicated by the dotted line in Figure 5(a). In Figure 5(b), the small dark dots were the silicon and carbon atoms. Although the silicon atoms were regularly arranged in the silicon substrate, as shown at the bottom of Figure 5(b), the arrangement of the atoms above the substrate was random, as shown at the center of Figure 5(b). Thus, the obtained silicon carbide film was considered to be amorphous.

Figure 6 shows the TEM image of the thin film obtained from the gas mixture of trichlorosilane gas and monomethylsilane gas at the flow rate of 0.06 and 0.05 slm, respectively, in hydrogen gas at $0.89 \mathrm{slm}$ at $600^{\circ} \mathrm{C}$ 


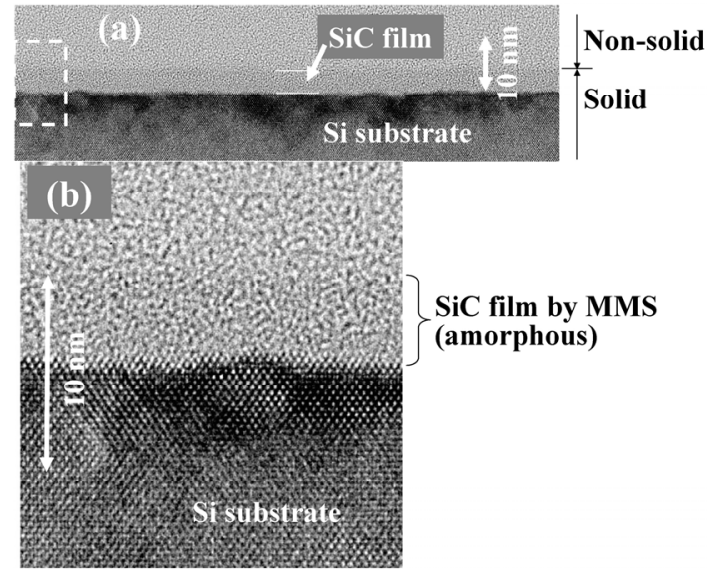

Figure 5. TEM image of silicon carbide thin film obtained from monomethylsilane gas at the flow rate of $0.05 \mathrm{slm}$ in hydrogen gas at $0.95 \mathrm{slm}$ at $600^{\circ} \mathrm{C}$ for $10 \mathrm{~min}$.

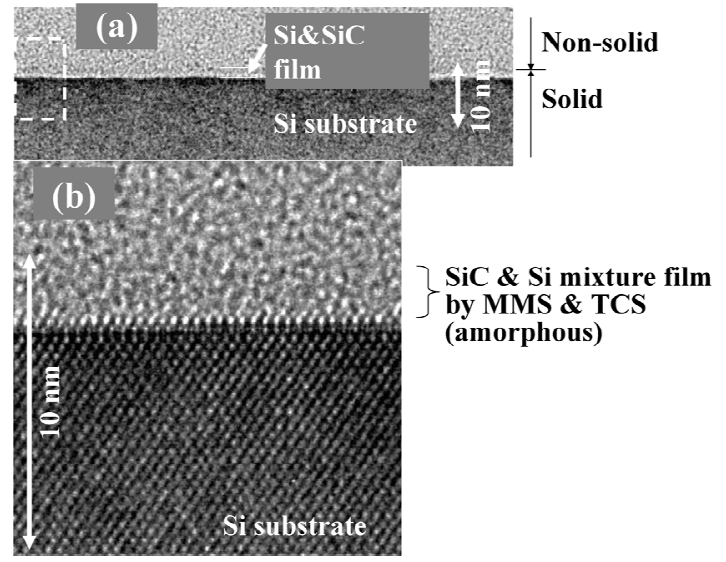

Figure 6. TEM image of the thin film obtained from trichlorosilane gas and monomethylsilane gas at the flow rates of 0.06 and $0.05 \mathrm{slm}$, respectively, in hydrogen gas at $0.89 \mathrm{slm}$, at $600^{\circ} \mathrm{C}$ for $10 \mathrm{~min}$.

for 10 min. Figure 6(a) shows the entire surface morphology of the obtained film. Taking into account the weak contrast to the non-solid region, the obtained film thickness was estimated to be about 2 - $3 \mathrm{~nm}$. Additionally, the film surface showed a smooth morphology. Figure 6(b) is a magnification of the area indicated by the dotted line in Figure 6(a). In this figure, the small dark dots were the silicon and carbon atoms. The silicon atoms were regularly arranged in the silicon substrate, as shown at the bottom of Figure 6(b). In contrast, the atoms above the substrate were randomly arranged, as shown at the center of Figure 6(b). Thus, the obtained film was considered to be amorphous. From these results, the obtained film was crystalline from trichlorosilane, amorphous from monomethylsilane and also amorphous from the mixed precursors.

Although the average film thickness of the crystalline silicon film, shown in Figure 4, could not be easily estimated due to its rough surface, it was estimated to be significantly less than $20 \mathrm{~nm}$. Taking into account that the density of silicon is about $74 \%$ of silicon carbide, the weight of the formed crystalline silicon film can be comparable to that of amorphous silicon carbide film in Figure 3. The mixed film of silicon and silicon carbide shown in Figure 6 was the thinnest in this study. Because the density of the obtained film is expected to be that between silicon and silicon carbide, the film weight shown in Figure 6 was considered to be the smallest in this study. Taking into account that the flow rate of monomethylsilane in Figure 6 was greater than that used in Figure 3 , this trend is considered to agree with that expected from the gradient in the LCM frequency, shown in Figure 3. Thus, the in-situ measurement using the LCM was considered to have the capability for predicting the actual trends in the film deposition behavior.

\subsection{Surface Process}

From the results obtained in this study, the surface process is briefly discussed using Figure 7, which is the schematic of the surface chemical process including trichlorosilane and monomethylsilane, taking into account the previous studies [7,9]. Pass (a) is an approach of trichlorosilane to chemisorbed monomethylsilane $\left(>\mathrm{SiHCH}_{3}\right.$, ">": two chemical bonds), and Pass (b) to chemisorbed trichlorosilane $\left(>\mathrm{SiCl}_{2}\right)$. Pass (c) is an approach of monomethylsilane to chemisorbed monomethylsilane $\left(>\mathrm{SiHCH}_{3}\right)$, and Pass (d) to chemisorbed trichlorosilane $\left(>\mathrm{SiCl}_{2}\right)$. Pass (e) is desorption of hydrogen, and Pass ( $\mathrm{f}$ ) is chlorine removal and hydrogen chloride production by hydrogen.

Because the obtained film from the mixed precursors

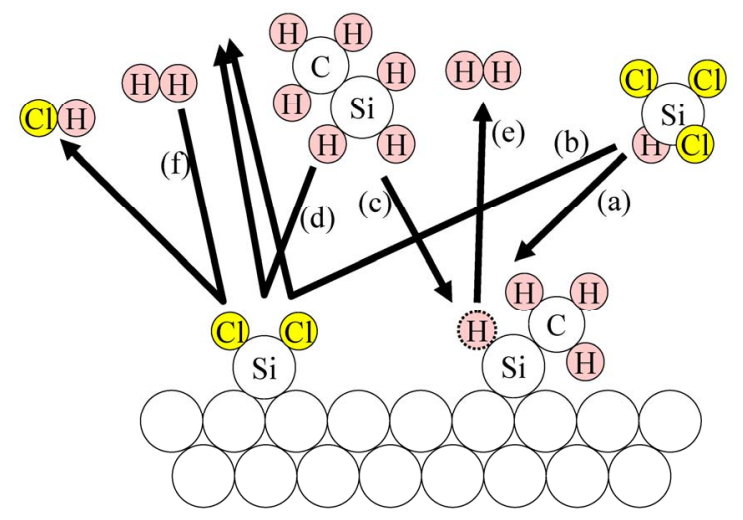

Figure 7. Schematic of surface chemical process. Approach of trichlorosilane (a) to chemisorbed monomethylsilane, and (b) to chemisorbed trichlorosilane. Approach of monomethylsilane (c) to chemisorbed monomethylsilane, and (d) to chemisorbed trichlorosilane. (e) is desorption of hydrogen, and (f) is chlorine removal and hydrogen chloride production by hydrogen. 
was amorphous for the flat surface, as shown in Figure 6, the major nature of the obtained film was considered to be classified as that of amorphous silicon carbide. Thus, the dominant chemical process is assumed to consist of Pass (c). Additionally, because the film thickness from the mixed precursors was a combination of that from each precursor, some influence of Pass (d) should be taken into account.

When the film deposition is performed with the single precursor of monomethylsilane, monomethylsilane gas continuously approaches the adsorbed $>\mathrm{SiHCH}_{3}$. Due to the low temperature, silicon and carbon atoms are nonregularly arranged over the surface to produce the amorphous film. This might be caused by the remaining hydrogen bonded with the carbon. Because the desorption of hydrogen atoms from carbon atoms requires a temperature higher than $1000^{\circ} \mathrm{C}[12,13]$, the regular arrangement of silicon and carbon atoms is disturbed by the remaining hydrogen atoms. Simultaneously, because the hydrogen atoms bonded with the silicon can be desorbed near $600^{\circ} \mathrm{C}$ [15], following Pass (e), the monomethylsilane gas can be chemisorbed on the silicon atoms [9] followed by hydrogen desorption.

During the film deposition using trichlorosilane added to monomethylsilane, the removal of chlorine atoms bonded with silicon atoms is necessarily performed by the chemical reaction with hydrogen [7], following Pass (f). Because the hydrogen removal process near $600^{\circ} \mathrm{C}$ is significantly slow, and because the chemical reaction of monomethylsilane with the chlorine atom of trichlorosilane is not easy [7], the chemisorbed trichlorosilane $\left(>\mathrm{SiCl}_{2}\right)$ is considered to remain for a long period at the surface to decrease the surface reaction rate. Thus, the entire surface reaction rate could be suppressed by adding trichlorosilane gas to monomethylsilane gas.

\section{Conclusion}

The langasite crystal microbalance (LCM) was applied for evaluating the thin film deposition from precursors, such as trichlorosilane, monomethylsilane and their mixture, at $600^{\circ} \mathrm{C}$, in order to evaluate the trends in the surface reaction and the deposition rate caused by various kinds of precursors. The film thickness trend obtained by the LCM was verified by the TEM. The weight of the crystalline silicon film from trichlorosilane gas was comparable to that of the amorphous silicon carbide film from monomethylsilane gas. The mixed film of silicon and silicon carbide from the gas mixture was the thinnest in this study. Because the film weight trend obtained by the LCM agreed with that by the TEM, the LCM is shown to be a convenient evaluation tool for the behavior of various film deposition. From the measurements in this study, the surface chemical process for the film deposition from a gas mixture of trichlorosilane and monomethylsilane is considered to be significantly suppressed by the chlorine atoms bonded with silicon in trichlorosilane. Because the thickness trend obtained by the LCM agreed with that by the TEM, the LCM is shown to be a convenient evaluation tool for the behavior of various film deposition.

\section{Acknowledgements}

The authors would like to thank Mr. Nobuyoshi Enomoto for his technical support.

\section{REFERENCES}

[1] A. M. Rinaldi and D. Crippa, "Silicon Epitaxy (Editor: D. Crippa, D. L. Rode and M. Masi)," Chapter 1, Academic Press, San Diego, 2001, p. 1. doi:10.1016/S0080-8784(01)80179-4

[2] K. Maeda, "VLSI \& CVD," Maki Shoten, Tokyo, 1997.

[3] D. Shen, H. Zhang, Q. Kang, H. Zhang and D. Yuan, "Oscillating Frequency Response of a Langasite Crystal Microbalance in Liquid Phases," Sensors and Actuators B, Vol. 119, No. 1, 2006, pp. 99-104. doi:10.1016/j.snb.2005.12.001

[4] M. Schulz, J. Sauerwald, H. She, H. Fritze and H. L. Tuller, "Defect Chemistry Based Design of Monolithic Langasite Structures for High Temperature Sensors," Solid State Ionics, Vol. 184, No. 1, 2011, pp. 78-82. doi:10.1016/j.ssi.2010.08.009

[5] H. Habuka and K. Kote, "Development of Reactive Surface Preparation for Room Temperature Silicon Carbide Film Deposition from Monomethylsilane Gas," Japanese Journal of Applied Physics, Vol. 50, No. 9, 2011, pp. 1-4. doi:10.1143/JJAP.50.096505

[6] H. Habuka and Y. Tanaka, "Langasite Crystal Microbalance Used for in-Situ Monitoring of Amorphous Silicon Carbide Film Deposition," ECS Journal of Solid State Science and Technology, Vol. 1, No. 2, 2012, pp. 62-65. doi:10.1149/2.006202jss

[7] H. Habuka, T. Nagoya, M. Mayusumi, M. Katayama, M. Shimada and K. Okuyama, "Model on Transport Phenomena and Epitaxial Growth of Silicon Thin Film in $\mathrm{SiHCl}_{3}-\mathrm{H}_{2}$ System under Atmospheric Pressure," Journal of Crystal Growth, Vol. 169, No. 1, 1996, pp. 61-72. doi:10.1016/0022-0248(96)00376-4

[8] H. Habuka, T. Otsuka and M. Katayama, "In Situ Cleaning Method for Silicon Surface Using Hydrogen Fluoride Gas and Hydrogen Chloride Gas in a Hydrogen Ambient," Journal of Crystal Growth, Vol. 186, No. 1, 1998, pp. 104-112. doi:10.1016/S0022-0248(97)00469-7

[9] H. Habuka, Y. Ando and M. Tsuji, "Room Temperature Process for Chemical Vapor Deposition of Silicon Carbide Thin Film Using Monomethylsilane Gas," Surface and Coatings Technology, Vol. 206, No. 1, 2011, pp. 15031506. doi:10.1016/j.surfcoat.2011.09.037

[10] H. Habuka, H. Ohmori and Y. Ando, "Silicon Carbide 
Film Deposition at Low Temperatures Using Monomethylsilane Gas," Surface and Coatings Technology, Vol. 204, No. 1, 2010, pp. 1432-1437. doi:10.1016/j.surfcoat.2009.09.044

[11] H. Habuka and Y. Ando, "Mechanism of Silicon Carbide Film Deposition at Room Temperature Using Monomethylsilane Gas," Journal of The Electrochemical Society, Vol. 158, No. 4, 2011, pp. H352-H357. doi:10.1149/1.3545071

[12] H. Habuka, M. Watanabe, Y. Miura, M. Nishida and T. Sekiguchi, "Polycrystalline Silicon Carbide Film Deposition Using Monomethylsilane and Hydrogen Chloride Gases," Journal of Crystal Growth, Vol. 300, No. 1, 2007, pp. 374-381. doi:10.1016/j.jcrysgro.2007.01.003
[13] H. Habuka, M. Watanabe, M. Nishida and T. Sekiguchi, "Polycrystalline Silicon Carbide Film Deposition Using Monomethylsilane and Hydrogen Chloride Gases," Surface and Coatings Technology, Vol. 201, No. 1, 2007, pp. 8961-8965. doi:10.1016/j.surfcoat.2007.04.023

[14] G. Sauerbrey, "Verwendung von Schwingquarzen zur Wägung dünner Schichten und zur Mikrowägung," Zeitschrift fur Physik, Vol. 155, No. 2, 1959, pp. 206-222. doi:10.1007/BF01337937

[15] K. Senthil, H. Nakazawa and M. Suemitsu, "Adsorption and Desorption Kinetics of Organosilanes at $\mathrm{Si}(001)$ Surfaces," Japanese Journal of Applied Physics, Vol. 42, No. 11, 2003, pp. 6804-6808. doi:10.1143/JJAP.42.6804 